International Journal of Computer Engineering and Technology (IJCET)

Volume 12, Issue 2, May-August 2021, pp. 1-10, Article ID: IJCET_12_02_001

Available online at https://iaeme.com/Home/issue/IJCET? Volume $=12 \&$ Issue $=2$

Journal Impact Factor (2020): 10.8630 (Calculated by GISI) www.jifactor.com

ISSN Print: 0976-6367 and ISSN Online: 0976-6375

DOI: 10.34218/IJCET.12.2.2021.001

(C) IAEME Publication

Scope Database Indexed

\title{
OPTIMIZATION OF QOS REQUIREMENTS FOR APPLICATIONS
}

\author{
Nadir K.Salih \\ Electrical and Computer Department, College of Engineering, \\ Buraimi University, Oman \\ Hanan Ahmed \\ Information Technology Department, Sudan University, Sudan \\ Nada A. Mohamed Nour \\ College of Computer Science and Information, \\ Princess Nourah Bint Abdulrahman University, KSA \\ Eshtiag A. Abd Elrhman \\ Deanship of Community Service and Continuing Education, \\ Jazan University, KSA
}

\begin{abstract}
QoS is critical and wide research area, it must applied for all fields, because any type of services presented for human achieve required quality to avoid disasters which may happened if there any defect in service. Sometime the disaster kill more people or cause other types of problem for them. For mentioned the measurements and validate the QoS is important activity before use a service, and the result of validation is accurate or not. The research is about the validity of QoS requirements for applications. It concern about the verification if the application satisfies user needs which specify inform of QoS requirements. The technique used to solve research problem is temporal logics; PCTL and CSL to represent QoS requirements of system inform of states using Markov chains, and then use PRISM model checker software to check the validity of requirements. The results of experiments explain which QoS requirements achieved and violated in procurement systems.
\end{abstract}

Key words: Quality of Services, Computation Tree Logic, Probabilistic Computation Tree Logic, Continuous Stochastic Logic, Markov Chains

Cite this Article: Nadir K.Salih, Hanan Ahmed, Nada A. Mohamed Nour, Eshtiag A. Abd Elrhman, Optimization of QoS Requirements for Applications, International Journal of Computer Engineering and Technology 12(2), 2021, pp. 1-10. https://iaeme.com/Home/issue/IJCET?Volume $=12 \&$ Issue $=2$ 


\section{INTRODUCTION}

Each service intended for customer must satisfy their needs and present service as required from the customers. The word service is a common, it include any type of service at all fields. The customer do not benefits from services if they have functional defects or failure in satisfy all users' needs, in addition to disaster which may happen if the services established without standard, this is common on all fields; industry, medicine, education etc. Sometimes small error kill more people especially in industry or medical field, and in marketing field cause more losses. For all these reason and to present safe services for customers. QoS be popular term because it means safety, satisfaction user needs and guarantee of specified services level [21]. So there must method for measure and verification from QoS, at each field QoS has different mechanism, and different factors or can say parameters must measure to evaluate QoS. So to decide about QoS firstly must specify what important parameters for a services and then choose the mechanism or technique to evaluation processes. Before that any service must has threshold which specify the level of quality, the threshold specified during negotiation between service provider and customers, the result of the negotiation called service level agreement (SLA).

The study of this research about validity of QoS requirements for application, the QoS requirements which need to validate was specified according to customer's satisfaction level required from application. The applications used as a cases study is a web applications developed to run for departments on ministry of justice. The technique used for validity by model checking. To represent the requirements the temporal logic PCTL and CSL was used. Model checker software PRISM applied to check the module of application and its QoS requirements.

The rest of the paper is structured as follows: Section 2 discusses the related work. Section 3 presents technique for proposed solution. Section 4 represents the solution model and case study. Section 5 demonstrates the analysis of result gained from experiments and section 6 include the conclusion and future work.

\section{RELATED WORK}

There are more studies about QoS, these studies different in techniques used to measure and the purpose which QoS evaluated. Here will present number of studies and their techniques and purpose of study which initiated in the following:

\subsection{Evaluated Services by Measure QoS}

Here the main purpose for QoS evaluation is to evaluate the service level. Each study use different technique in evaluating QoS. In [1] they used QoS strategy to compare pre-QoS requirements which represent expected QoS requirement with post-QoS requirements which represent actual QoS requirements to identify what are parameters responsible for delay problem. Authors In [2] they proposed key performance indicators as metrics for decide if the service present user requirements, these keys using counters to evaluate performance for TETRA networks, each QoS parameter evaluated using group of counters. In mobile edge technology to choose service which satisfy user requirements must predict QoS they use collaborative filtering based on user mobility to accurately predict QoS when specify mobile edge to be invoke for serve user[3]. In cloud applications built on service-oriented architectures when cloud environments change must keep QoS guarantee, to achieve this task. The researchers in [4] they used adaptive matrix factorization to perform online QoS prediction for candidate services. In [18] the researcher to accurate QoS prediction for web service they proposed probabilistic matrix factor model, this model depend on users properties and their physical neighbors' performance, the result of experiment explain that the proposed method performed better than state-of-the-art approaches. 


\subsection{Measure QoS for Select the Best Services}

To choose between services has the same functionality, need to measure QoS for each one to specify the best one according to user requirements. To select best web service from many services available with the same functionality [5] they use hidden Markov models to predict QoS for a web service, because it is probabilistic models allow building behavior model for web services for near future, so can predict QoS for it. To select cloud computing service, the QoS is important factor, therefore QoS prediction for cloud service support user to select most suitable service. In [6] they used Bayesian network model of QoS prediction for cloud services to consider the three layer structure of cloud computing model, because it effect on QoS prediction. In [7] they propose trustworthy service selection approach integrating cloud model and interval number theory of potential users. They use QoS evaluation to comparison between the trustworthiness cloud services and then transform to interval number based on cloud model to create set of trustworthy services, and then use ranking system to select the most trustworthy service. In[8] proposed ranking system to select web services depend on the functional relevance, user behavior, QoS and service usage factor to accurate the result they consider consumption history. QoS preferences and QoS constraint were explored at large which is considered. The result is better in satisfying users from existing ranks system. Continuing in field of how to fit suitable services for consumer, in [20] they proposed QoS architecture based on the set of attributes which considered when building concrete grid network for provide service in this framework must specify QoS module that offer best QoS level, the QoS module seeking for best fit of services provider for consumer.

\subsection{Measure QoS for Composition}

If the user need to certain services not available by one application, but each feature of service available cross single service, to solve this problem composition more than one application compose together to provide required features, the optimal composition is choose according results of QoS measurements. In [9] dynamically composition web services depend on QoS parameter to select the most relevant using genetic algorithm. In [10] they addressed the problem of finding optimal QoS available services for composition, it consider is optimization problem, they presented meta-heuristic bio-inspired to QoS-aware web service composition. It based on elephant herding optimization (EHO) algorithm. The evaluation of proposed solution explains that it better than existing algorithm. In [11] the authors proposed service selection approach based on QoS prediction to composition optimal services. To predict QoS for web service they consider historical QoS information as a time series and predict QoS values using the autoregressive integrated moving average model to provide more accurate QoS attribute values. In services composition when large scale of services needs to search in to find the optimal services according to user requirement, the problem is time consuming. [12] To solve this problem they use method based on modified algorithm of graph coloring. they implement MGC-TOP K and MGC-K. In [19] they addressed the problem of composition by reducing optimal QoS that satisfy the customer requirement in big services environment. They proposed novel MapReduce based evolutionary algorithm with guided mutation considering QoS. The evaluation of proposed mechanism that is outperforms other methods.

\section{METHODOLOGY AND TECHNIQUES}

The proposed solution for research problem depends on probabilistic techniques because the solution is verification for system QoS requirements, and the probabilistic technique specification has ability to achieve the solution according to require. Probabilistic techniques are techniques can be used to modeling, specifying, and proving timing properties of real-time 
systems. The one of the probabilistic technique is temporal logics which used in the proposed solution:

\subsection{Temporal Logic}

Temporal logics allows you to specify the behavior of systems in terms of logical formulas, including time constraints, events, and relationships between the them [13]. The temporal logics were mathematically founded, so these techniques facilitate modeling, specifying, and proving timing properties of real-time systems, also have different capabilities for the specification, validation, and verification [14]. Temporal logics have multi types; in our model will use combination branching time temporal logic called computation tree logic (CTL) and probabilistic temporal logic, Probabilistic Computation Tree Logic (PCTL), and another one also is computation tree logic is continuous stochastic logic (CSL). To represent system behaviors in form of events and time constrain used Markov chains. After build system model then used model checker software to validate the model. Below demonstrate parts of technique.

\subsubsection{Markov Chains}

Markov chains can characterizes as follow: each system have set of states $S=$ $\left\{\mathrm{s}_{1}, \mathrm{~S}_{2}, \mathrm{~s}_{3}, \ldots \ldots \ldots \mathrm{s}_{\mathrm{n}}\right\}$ the set is state space, the transition from one state to another is called step, the probability of what is the next step called transition probability. The Markov chain is type of Markov process in either discrete or continuous time.

\subsubsection{PCTL}

PCTL is used to represent properties of application at discrete state; this means the application state change at deferent point of time [15], one time unit corresponds to one transaction along an execution path. Discrete time Markov chain (DTMC) interpreted PCTL formulas. The PCTL has state formulas and path formulas, state formulas represent states properties while path formula represents paths properties (sequence of states).

\subsubsection{CSL}

Is a powerful logic for expressing quantitative time-bounded constrained reachability properties. CSL as PTCL has state formula and path formula. To represent CSL the continuous time Markov chain (CTMC).

\subsubsection{Probabilistic Model Checking}

Probabilistic Model Checking is an automatic model-based verification approach used to analyze probabilistic system and explores all system executions, the analysis will use in correctness, performance and reliability of system [16].at the study used software model checker PRISM. It is an open source probabilistic model checker developed initially at the University of Birmingham and now at the University of Oxford. It is supports three probabilistic models: discrete-time Markov chains (DTMC), continuous-time Markov chains (CTMC) and Markov decision processes [17], these models are described in the PRISM modeling language, a relatively simple, state-based language and properties are specified in a logic which incorporates LTL, PCTL, CSL.

\subsubsection{Probability Assignment Techniques}

The probability of an event is a numerical measure of the chance that this event will occur. The probability value of event must calculate according to following conditions: 
1. A given statistical experiment has at least two experimental outcomes. Depending on the problem being analyzed, neither some of the outcomes, nor even all of them could be events of statistic interest

2. The probability of any event $e_{i}$ if denote it $P\left(e_{i}\right)$ must lie in range between 0 and 1: $0 \leq P\left(e_{i}\right)$ $\leq 1$

3. The sum of the probabilities of all $\mathrm{n}$ experimental outcomes equals $1: \mathrm{P}\left(\mathrm{e}_{1}\right)+\mathrm{P}\left(\mathrm{e}_{2}\right)+$ $\mathrm{P}\left(\mathrm{e}_{3}\right)+\ldots . .+\mathrm{P}\left(\mathrm{e}_{\mathrm{n}}\right)=1$.

To assign probability values there three approaches of assigning, those approaches depend on the nature of the information that is available, below will mention details about each approach:

1-The classical method for assigning probability:

If probabilities of the experimental outcomes satisfy the following assumptions:

a)The probabilities of all of the outcomes are known in advance

b) The outcomes are equal probable (all the outcomes are equally likely). Then probability of each of them outcomes is $1 / \mathrm{n}$.... then we can deal with so called classical method of assigning probabilities.

2-Relative Frequency method of assigning probability:

When the assumption that the outcomes of a statistical experiment are known in advance and are equally likely is not satisfied, the estimation of probability for events of interest can be done by using past statistics.

3-subjectivemethod:

This method is used when the assumptions used in the classical method are not applicable and the past statistics that can be used for the relative frequency method are unavailable. In such a situation, the basis for assigning probability to experimental outcomes is previous business experience, belief, and even feeling. Since this method relies on individual judgmental, it is highly subjective. Nonetheless, the method is quite common in the absence of any information. Later, when the information becomes available, the assigned probability can be revised. In this study was using the third approach.

\section{SOLUTION MODEL}

To test proposed solution take two systems as a case study, they are web application. After specify case study describe QoS requirements for each one as a probability and then transfer the systems to states using Markov chains, these states which need to validate their quality, so the requirements probability representing in systems states, the QoS requirements representing in Markov chain according to time properties; DTMC or CTMC. Then use PRISM as a software model checker to validate if the system satisfies QoS requirements. Below demonstrate the solution in more details:

\subsection{Case Study no.1: Procurement System}

The system was developed for procurement department on ministry of justice to store and monitor the processes of order until served and archive. Figure.1 explains the system processes flowchart: 


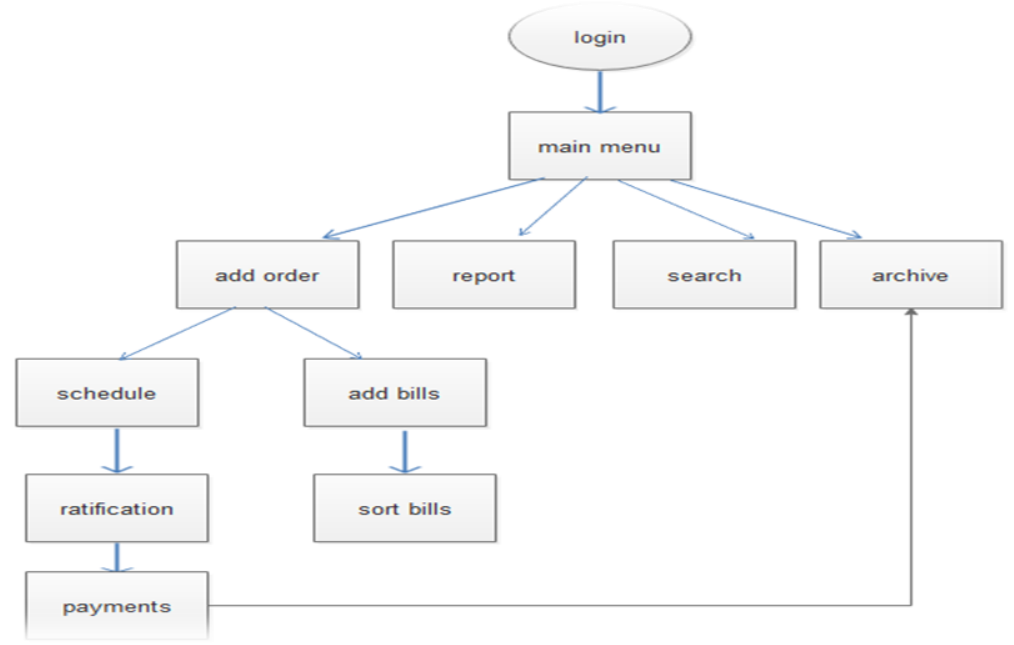

Figure 1 Procurement process flowchart

Then specify system QoS requirements which need to verification:

\section{Security requirements:}

$\mathrm{R}_{1}$ : The probability $\mathrm{P}_{1}$ of lock user account if he try more than 3 times $\mathrm{P}_{1=} 0.02$.

$\mathrm{R}_{2}$ : The probability $\mathrm{P}_{2}$ of user $\log$ out if he take time of rest without transaction more than 60 seconds $\mathrm{P}_{2} \leq 0.5$.

\section{Process execution time requirement:}

$R_{3}$ : The probability $P_{3}$ of request of site failed if it takes more than 120 seconds $P_{3} \leq 0.3$.

$\mathrm{R}_{4}$ : The probability $\mathrm{P}_{4}$ of any process will drop if it takes more than 10 seconds $\mathrm{P}_{4} \leq 0.2$.

\section{Availability requirements:}

$\mathrm{R}_{5}$ : The probability $\mathrm{P}_{5}$ of connection for application server failed $\mathrm{P}_{5}=0.1$.

$\mathrm{R}_{6}$ : The probability $\mathrm{P}_{6}$ of connection for database server failed $\mathrm{P}_{6}=0.02$.

According requirements use DTMC to represent discrete time states and CTMC for continuous or bounded time. Below figure for each one:

The requirements $\mathrm{R}_{1}$ and from $\mathrm{R}_{5}$ to $\mathrm{R}_{6}$ represented in DTMC

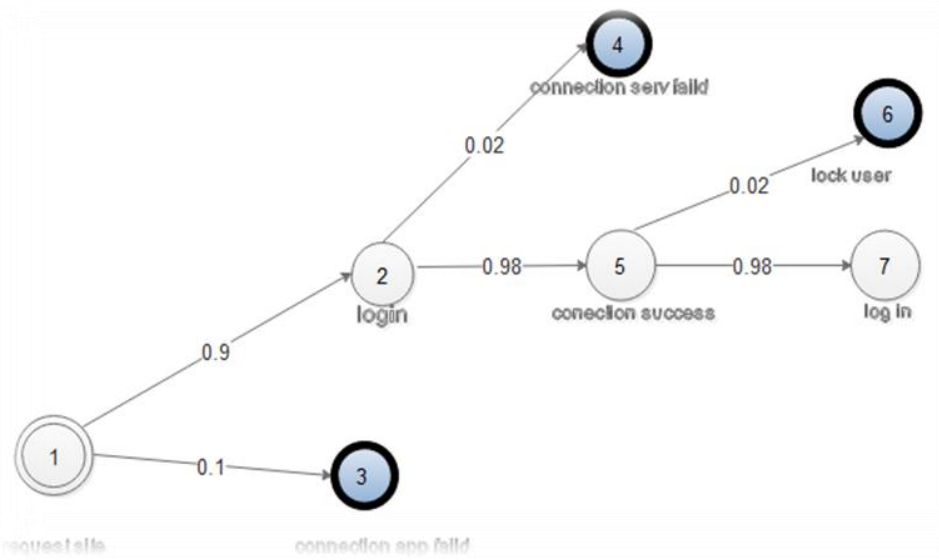

Figure 2 Requirements of DTMC 
The requirements can be translated to PCTL as follow:

- $\quad P_{\geq 0.1}=[\diamond s=3]$ The probability of reaching state called connection application failed is greater than or equal 0.1 .

- $P_{\geq 0.02}=[\diamond s=4]$ The probability of reaching state called connection serv failed is greater than or equal 0.02 .

- $P_{\geq 0.02}=[\diamond s=6]$ The probability of reaching state called lock of user is greater than or equal 0.02 .

The requirements from $\mathrm{R}_{2}$ to $\mathrm{R}_{4}$ represented in CTMC

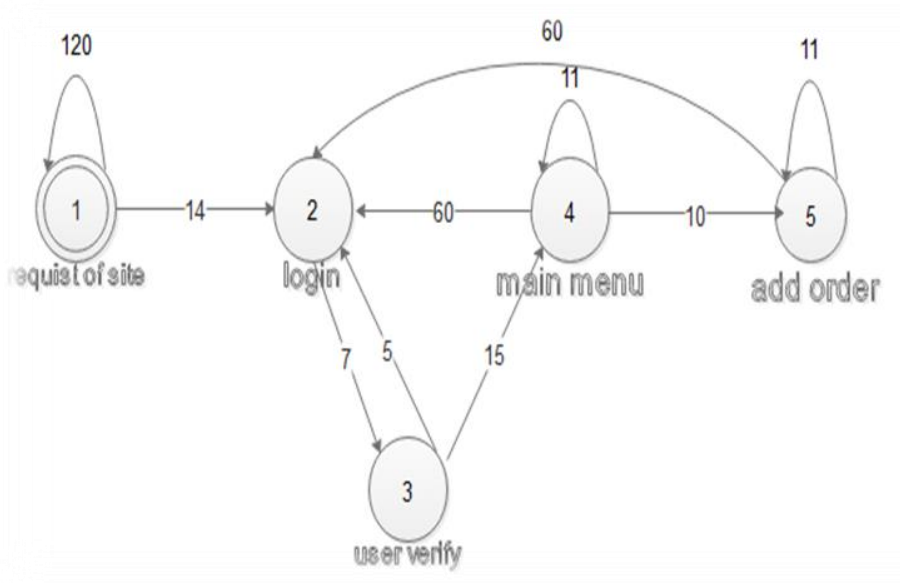

Figure 3 Requirements of CTMC

The requirements can be translated to CSL as follow:

- $P_{\leq 0.5}=\left[0^{\leq 60} s=2\right]$ The probability of system back to login state within $60 \mathrm{~s}$ is less than or equal 0.5

- $P_{\leq 0.3}=\left[{ }_{0} \leq 120 s=1\right]$ The probability of system stay at request of site within $120 \mathrm{~s}$ is less than or equal 0.3

- $\quad P_{\leq 0.2}=\left[{ }^{\leq 10} s=5\right]$ The probability of system drop any of process and back to previous within 10 s less than is less than or equal 0.2 .

To validate system QoS requirements use model checker PRIM: As in figure 4 Transfer DTMC to modules according to figure 2 mentioned above.

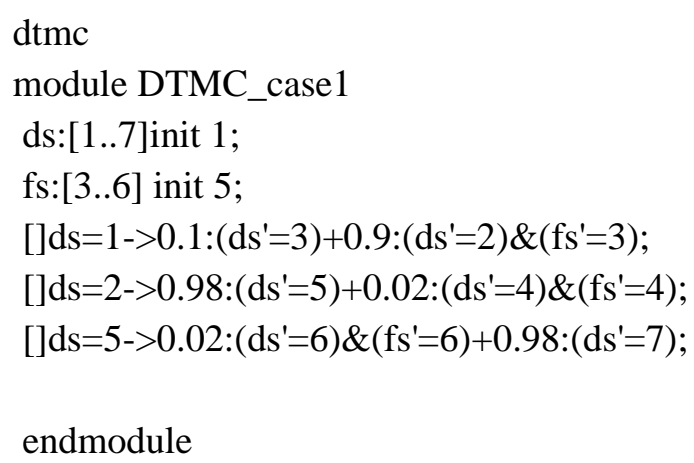

Figure 4 DTMC module representation 
To represent QoS requirements and system states in PRISM use model language, and then run the module to gain result of experiments, I recurrent the experiment for assurance from the result and generate graph from experiment represent validity properties value.

The result of experiments is:

- Probability of connection to application failed equal 0.1.

- Probability of connection of server failed equal 0.0180 .

- Probability of user locked equal 0.01764 .

And graph generated is:

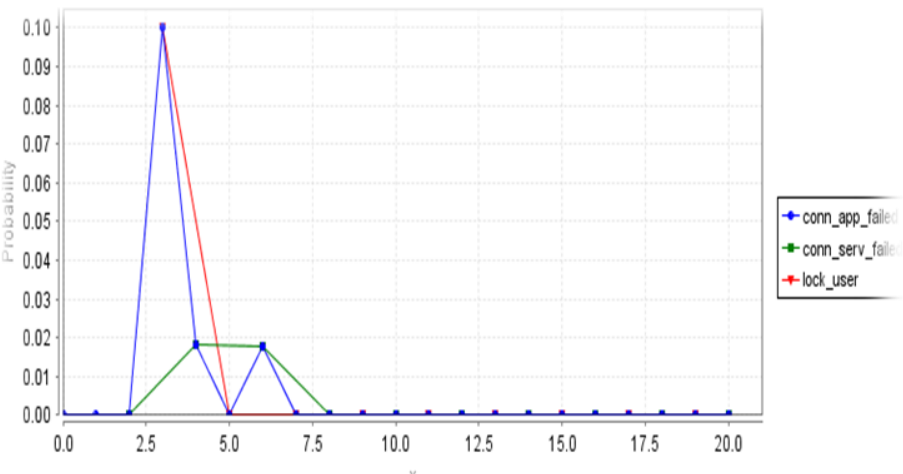

Figure 5 DTMC module experiment result

To validate system QoS requirements use model checker PRIM: As in figure 6 Transfer The module represent CTMC is below according to figure 3 mentioned above

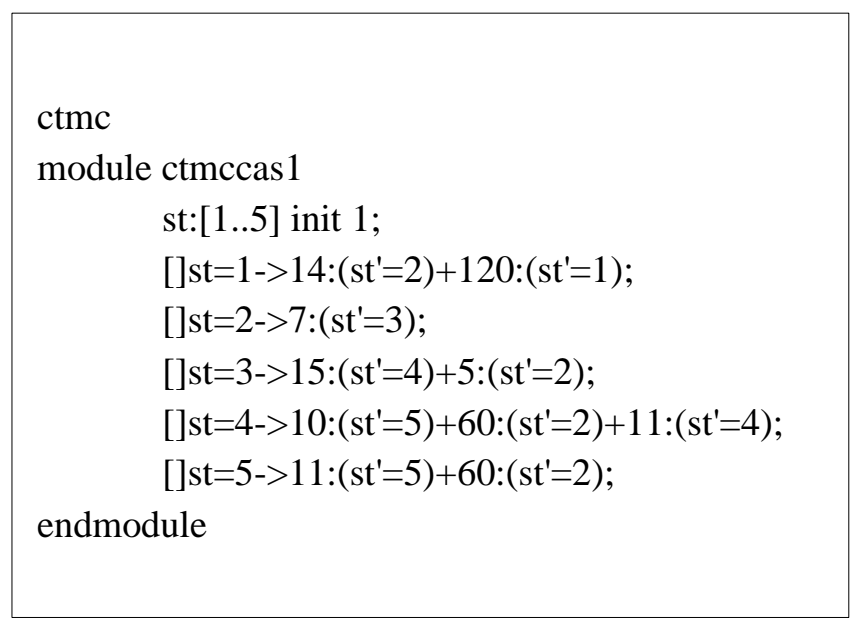

Figure 6 CTMC module representation

The experiment result is:

- Probability of loading time out equal 1.

- Probability of session time out equal 1.

- Probability of drop process equal 1.

And graph generated is: 


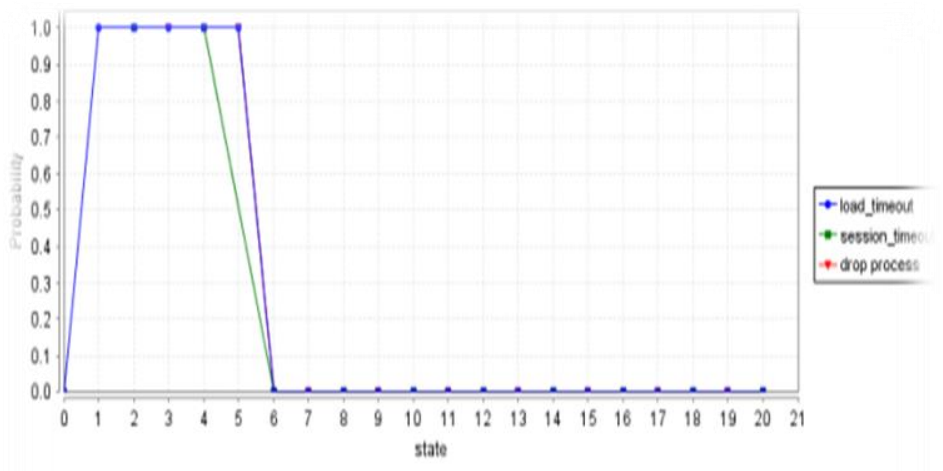

Figure 5 CTMC module experiments result

\section{RESULT ANALYSIS}

The result obtained from module experiments explain that, the requirements represented as DTMC achieve the required level of QoS because the value of probabilities resulted less than the proposed probabilities, these result mean the system achieve QoS requirements, but the requirements represented as CTMC violated, because the value of probabilities is greater than proposed probabilities, so the system must redevelop to achieve all user satisfaction level.

\section{CONCLUSION}

The study was initiated for validity of QoS requirements for application using probabilistic model checking, this technique is more effective in verification than simulation because is performed automatically and detect QoS violation.

\section{REFERENCES}

[1] P. Calduwel Newton and L. Arockiam, A Quality of Service Performance Evaluation Strategy for Delay Classes in General Packet Radio Service, International Journal of Advanced Science and Technology 2013, pp 91-98.

[2] José D. Luis Delgado, Jesús Máximo Ramírez Santiago, Key Performance Indicators For QOS Assessment In Tetra Networks, International Journal of Mobile Network Communications \& Telematics (IJMNCT) 2013.

[3] Shangguang Wang, Yali Zhao, Lin Huang, Jinliang Xu1, Ching-Hsien Hsu, QoS Prediction for Service Recommendations in Mobile Edge Computing.

[4] Jieming Zhu, Pinjia He, Zibin Zheng, and Michael R. Lyu, Online QoS Prediction for Runtime Service Adaptation via Adaptive Matrix Factorization, IEEE Transactions on Parallel And Distributed Systems 2017.

[5] Daniel G. Canton-Puerto, Francisco Moo-Mena, and Víctor Uc-Cetina, QoS-Based Web Services Selection Using a Hidden Markov Model, Journal of Computers 2017.

[6] Pengcheng Zhang, Qing Han, Wenrui Li, Hareton Leung, and Wei Song, A Novel QoS Prediction Approach for Cloud Service Based on Bayesian Networks Model, IEEE International Conference on Mobile Services 2016, pp 111 to 118. 
[7] MA Hua, HU Zhigang and CAI Meiling, Trustworthy Service Selection Integrating Cloud Model and Possibility Degree Ranking of Interval Numbers, Chinese Journal of Electronics, 2017 pp 1177 to 1183.

[8] R. Sarala, P. Manisha, Mukku Vineesha, and G. Indumathy, A Consumption History and QoS based Web Service Ranking Technique, International Conference on Emerging Innovation in Engineering and Technology 2017, pp 15 to 20.

[9] Kirit J. Modi and Sanjay Garg, Dynamic Web Services Composition using Optimization Approach, IJCSC.2015, pp 285-293.

[10] Samia Sadouki Chibani, Abdelkamel Tari, Elephant Herding Optimization for Service Selection in QoS-Aware Web Service Composition, World Academy of Science, Engineering and Technology International Journal of Computer and Information Engineering 2017, pp 1116 1120 .

[11] Yan Guo, Shangguang Wang, Kok-seng Wong and Myung Ho Kim, Skyline Service Selection Approach based on QoS Prediction, Int. J. Web and Grid Services 2016.

[12] Sepideh Sheivandi and Sima Emadi, Automatic Service Composition Based on Graph Coloring, Journal of Advances in Computer Research 2018, pp 91-102.

[13] Savas Konur, Real-time and Probabilistic Temporal Logics: An Overview November 2008.

[14] Chanyeol Yoo, Robert Fitch and Salah Sukkarieh, Probabilistic Temporal Logic for Motion Planning with Resource Threshold Constraints.

[15] Daniel Wagner, Finite-State Abstractions for Probabilistic Computation Tree Logic, Imperial College London, Department of Computing 2011.

[16] Marta Kwiatkowska ,Gethin Norman and David Parker, Quantitative analysis with the probabilistic model checker PRISM, QAPL 2005.

[17] Marta Kwiatkowska, Gethin Norman, and David Parker, PRISM: Probabilistic Symbolic Model Checker.

[18] Fei Peng, Xuewen Zeng, Haojiang Deng and Lei Liu, The QoS Prediction of Web Service with Location Information Ensemble, Journal of Software, VOL. 9, NO. 5, MAY 2014 (page from 1210 to 1216$)$.

[19] Chandrashekar Jatoth, G.R. Gangadharan, Ugo Fiore, Rajkumar Buyya, QoS-aware Big service composition using MapReduce based, Future Generation Computer Systems July,2017. Evolutionary algorithm with guided mutation

[20] Christos Chrysoulas, and Maria Fasli, Towards an adaptive SOA-based QoS \& DemandResponse Provisioning Architecture for the Smart Grid, Journal of Communications Software And Systems, VOL. 13, NO. 2, June 2017,pp.77-86.

[21] Nadir K. Salih, Abdel-hafiz A. Khoudour, Mawahib S. Adam, Samar M. Hassen "Autonomic Computing Architecture by Self-defined URI" International Journal of Computer Trends and Technology 68.3 (2020):1-6.2020 